\title{
Study of heavy metals effect in response to linum seed germination
}

\author{
Rajesh Kumar Jain
}

SIRDA Group of Institutions, Sunder Nagar, H.P, India. E-mail: duggarjain@yahoo.co.in, rajmeerutcity@gmail.com.

\author{
Accepted 11 October, 2011
}

\begin{abstract}
The aim of the present study is to develop phenotypically stable varieties for those soils that are contaminated with mercury or cobalt. This is a novel report about their ability to grow in the contaminated soil. The benefit of this technology is the potential for low cost remediation. Highly significant differences have been observed among the varieties of Linum usitatissimum for all the characters in all the treatment or environment. There has been considerable amount of variability for all the characters in all the treatments. The $10^{-5} \mathrm{M} \mathrm{HgCl}_{2}$ treatment shows the inhibitory effect in all the varieties and there is no further seedling establishment after seed germination. The highest toxic effect has been observed for the seedling vigour and seed vigour index. The $10^{-5} \mathrm{M} \mathrm{HgCl}_{2}$ concentration is the last limit of tolerance in plants while $10^{-4} \mathrm{M} \mathrm{CoCl}_{2}$ could be the last tolerance limit and there may be a specific gene in plants which monitor the toxicity level or tolerance capacity. The maximum magnitude of positive correlation coefficient has been found between seedling fresh and dry weight and negative for 1000 seed weight and germination rate index. The seedling length exerts maximum positive direct effect on seedling vigour followed by absolute seedling water content.
\end{abstract}

Key words: Linum, seed germination, $\mathrm{CoCl}_{2}, \mathrm{HgCl}_{2}$, variability, correlation, path.

\section{INTRODUCTION}

Among all the oil seed crops, linseed (Linum usitatissimum L.) ranks fourth in importance in term of area as well as production. It is cultivated in almost all the countries of the world. It is chiefly grown as a fibre crop in European and other temperate countries while in India it is exclusively cultivated as oil seed crop. Heavy metals contamination has been recognized as a major environmental concern due to their pervasiveness and persistence. These heavy metals are not bio-degradable, hence there is a need to develop such a remediation technique, which should be efficient, economical and rapidly deployable in a wide range of physical settings. It is also necessary to integrate different areas, such as biology, phytogeography, soil sciences and even anthropology to get a more dynamic view of the problem. Such changes might add significant new information to our knowledge on the subject. Elevated levels of heavy metals in contaminated soils are widely spread and concerns have been raised over the potential risks to humans, animals and agricultural crops. As a rule, heavy metal has a negative effect on the growth of organisms as it can greatly depress their numbers. Contamination of wastewater with high rates of heavy metals caused a significant decrease in biodiversity. Heavy metal contamination affects the biosphere in many places worldwide (Cunningham, et al., 1997; Raskin and Ensley, 2000; Meagher, 2000). Metal concentrations in soil range from less than $1 \mathrm{mg} / \mathrm{kg}$ (ppm) to high as $100,000 \mathrm{mg} / \mathrm{kg}$, whether due to the geological origin of the soil or as a result of human activity (Blaylock and Huang, 2000). Excess concentrations of some heavy metals in soils have caused the disruption of natural aquatic and terrestrial ecosystems (Gardea et al., 1996; Meagher, 2000). There is a great lack of knowledge defining the precise quantitative limits of tolerance that is the actual dosage level at which a chemical is toxic and the point beyond which no further adaptation can be achieved by a species (Dickinson et al., 1991). There seems to be no work on heavy metal toxicity in Linum except the one of Tkachuk and Kuzina (1972), who reported the mercury levels in flax seeds.

Heavy metals are included in the main category of 
environmental pollutants as they can remain in the environment for long periods; their accumulation is potentially hazardous to humans, animals and plants (Benavides et al., 2005; Gratão et al., 2005a). In the last few decades a dramatic, worrisome increase in contamination of the environment, including soil, air and water. It would appear that humans are the only ones to blame, because anthropogenic activities are the main source of the pollution that is causing the contamination (Gratão et al., 2005a). It is quite obvious from the studies carried out along the years that heavy metals have adverse effects on plants as well as animals and their productivity, although some metals are essential for plant growth in small quantities (Gomes et al., 2006). The accumulation of metals in plants is particularly important because some species have been characterized as hyper accumulators. They may be used, as along with specifically designed transgenic plants, in phytoremediation (Cherian and Oliveira, 2005; Eapen and D'Souza, 2005; Gratão et al., 2005b). Moreover, the use of bio-remediation techniques (Lynch and Moffat, 2005) has been replacing whenever possible the traditional engineering approaches. Such a situation has led to investigations on a wide range of aspects related to heavy metals. For instance, there has been intensive research on metals in soil related to plant nutrition, general effects on plant metabolism, tolerancesusceptibility, and environmental effects as well as on how contaminated areas can be reclaimed (Gratão et al., 2005a; Pilon, 2005; Taulavuori et al., 2005). In first few years of the 21 st century a boom in the research in defense system in response to heavy metal-induced stress in plant tissues. It is quite difficult to say exactly which specific aspects have received most attention.

There is still plenty for such analyses; however, feel that along with such re-search, other approaches should now be included to consolidate the work on stress induced by metals, or even other stresses. For instance, few has been published on research related to proteomics, metabolomics, metallomic and metalloproteins (Garcia et al., 2006). Molecular techniques, such as northern blots and PCR, which have been used to detect the level of gene expression, have been available for quite some time; however, they still do not predominate in this area; their importance in elucidating biochemical problems is undeniable. Such approaches are much expensive and can not be adopted rapidly by researchers working on heavy-metal effects on plants.

Currently, cleanup processes of heavy metal pollution are expensive and environmentally destructive (Nanda et al., 1995; Moffat, 1995; Meagher, 2000). Recently, scientists and engineers have started to generate costeffective technologies that include the use of microorganisms, biomass, and live plants in the cleaning process of polluted areas (Miller, 1996; Boyajian and Carreira, 1997; Dushenkov et al., 1997; Ebbs and
Kochian, 1998; Wasay et al.,1998; Gardea et al., 1996). Some heavy metals at low doses are essential micronutrients for plants, but in higher doses they may cause metabolic disorders and growth inhibition for most of the plants species (Fernandes and Henriques, 1991; Claire et al., 1991). Researchers have observed that some plants species are endemic to metalliferous soils and can tolerate greater than usual amounts of heavy metals or other toxic compounds. Evolved tolerance to toxic concentrations of heavy metals in plants inhabiting spoil heaps of mines is a well known phenomenon that has been the subject of much research in the last two decades. These plants are useful models for studying processes involved in the early stages of the speciation of edaphic endemics. Consequently, the information on variance, genetic variability, correlation coefficient and path coefficient is not available. Recent work has revealed the importance of several phenomena in the differentiation of tolerant populations, including natural selection, founder effects and 'hitch-hiking' .The present study is an endeavour in this direction.

\section{MATERIALS AND METHODS}

The experiments were carried out in the laboratory at room temperature. The effect of two heavy metals that is cobalt and mercury was recorded on the ten varieties of $L$. usitatissimum namely TLP-1, RLC-29, LC-54, LC-185, T-397, Kiran, Nagarkot, Neelum, Shubra and Gaurav with the help of unsoaked and soaked control experimental sets. For the control set, the tap water was taken. For heavy metal sets, $10^{-5}, 10^{-6}$ and $10^{-7}$ molar concentration solution of both the heavy metals were prepared in tap water. The experiments were conducted in glass Petri dishes having four replications. Each replication has 10 seeds. The seeds were imbibed in different concentrations of heavy metals along with control one for $24 \mathrm{~h}$ before sowing. After $24 \mathrm{~h}$ these seeds were continuously washed with running tap water and transferred to Petri dishes having wetted pads of same concentrations. The solution was applied in such a manner that the pads and seeds always remain wet. The visual emergence or protrusion of radicle was taken as the criteria for germination. The seed germination test was carried out according to rules laid down by International Seed Testing Association (1976). The percentage of seed germination was recorded till the fifth day after sowing. The germination rate index has been calculated with the help of modified method of Srivastava and Sareen (1972) and Heydecker (1973) using the following formula:

Germination rate index $=4(5 g+4 g+3 g+2 g+g)$

$\mathrm{g}=$ The number of germinated seed after each $24 \mathrm{~h}$.

The radicle and hypocotyl length has been measured from 5th to 7th day after sowing. The organ elongation rate has been calculated by Heydecker (1973) modified method.

(Mean length of the organ / Days to first count +++ Mean increase in length Days to last count)

The seedling length has been measured on seventh day after sowing. The cotyledonary area that is, length and breadth of cotyledons has been measured on seventh day of sowing. At the time of termination of experiments, ten seedlings were weighed and the same were dried in a hot air oven at $60^{\circ} \mathrm{C}$ for $24 \mathrm{~h}$ for obtaining the fresh and dry weight of seedlings. The absolute and specific 
seedling water content was calculated with the help of following formulas.

Absolute seedling water content $=$ seedling fresh weight - seedling dry weight.

Specific seedling water content $=$ Absolute seedling water content $/$ Seedling dry weight.

The seedling vigour and seed vigour index has been calculated with the help of the formula given by Abdulbaki and Anderson(1973).

Seedling vigour $=$ Percentage germination $x$ Seedling length Seed vigour index $=$ Percentage germination $x$ Mean dry weight The tolerance index (T.I.) was estimated by Wilkins (1978) formula. T.I. $(\%)=$ Organ growth in solution with metal / Organ growth in solution without metal.

The toxicity level was determined with the help of following formula

Toxicity level $(\%)=100$ - Tolerance index

The data thus collected were subjected to the following statistical and biometrical analysis. The analysis of variance was calculated by Panse and Sukhatme (1961) formulae. Phenotypic coefficient of variance (P.C.V.), genotypic coefficient of variance (G.C.V.), and heritability in broad sense were calculated using the formulae as suggested by Burten (1952). The expected genetic advance at 5\% intensity of selection differential was calculated by the Johanson et al. (1955) formulae and genetic advance as in percentage of mean according to Allard (1960) formula. The genotypic and phenotypic correlation coefficient among all characters under/study was estimated as given by Searle (1961). The direct and indirect effects were estimated in path coefficient analysis as suggested by Dewey and Lu (1959). Simmonds (1962) states that the range of variability depends upon the selection pressure under domestication that is, upon the system of agriculture. He further states that the imposition of new norms of selection, allowing the survival of only favored genotypes and variability is destroyed at a considerable rate. The rate and magnitude of variability depends upon factors such as system, growth habit and population size. We classified the G.C.V., P.C.V., heritability and genetic advance into high, moderate and low magnitudes for our convenience for the presentation and analysis of results.

However, there is no such particular criteria for the classification of G.C.V., P.C.V. and G.A. except in case of heritability where Robinson (1966) has given such range. In the present communication, we have considered the following range of G.C.V., P.C.V., heritability and genetic advance. The magnitude of G.C.V. and P.C.V. up to $10 \%$ as low, 10 to $20 \%$ moderate and above the $20 \%$ considered as high. The magnitude of heritability in broad sense has been considered as low below the 0.3 , between 0.3 to 0.6 as moderate and above 0.6 as high. The magnitude of G.A in percentage of mean up to $7 \%$ as low, between 7 to $15 \%$ as moderate and above $15 \%$ as high.

\section{RESULTS AND DISCUSSION}

Seed germination and seedling establishment are the two most important events in the life cycle of plants. Heavy metal pollution has created a great ecological crisis. Modern civilization introduces a wide range of pollutants to the atmosphere through various anthropogenic activities such as industry, mining, transportation, etc.

Despite the fact that, it is almost impossible to visualize a soil without trace levels of heavy metals and most of the heavy metals are essential elements for living organisms, but their excess amounts are generally harmful to plants, animals and human health (Azevedo and Lea, 2005; Jarup , 2003). Currently, contamination of soil in cultivated fields with toxic heavy metals has emerged as a new threat to agriculture (Singh et al., 2007). The present study reveals that there was no further growth after germination in $10^{-5} \mathrm{M} \mathrm{HgCl}_{2}$ concentration. All characters in all the varieties of $L$. usitatissimum showed significant differences in different environments except character like absolute seedling water content in unsoaked control environment (Table 1). The error variance is higher than their G.C.V. and P.C.V. for the characters like germination rate index, seedling vigour and vigour index in all the concentrations or environments. The percentage germination also shows similar results in unsoaked control, soaked control, $10^{-5}$ $\mathrm{M}, 10^{-6} \mathrm{M}$ and $10^{-7} \mathrm{M} \mathrm{CoCl}$. These findings indicate that these characters are mostly influenced by environment.

The percentage germination in $10^{-6} \mathrm{M}, 10^{-7} \mathrm{M} \mathrm{CoCl}_{2}$ and $10^{-6} \mathrm{M} \mathrm{HgCl}_{2}$ shows lower error variance value then their respective G.C.V. and P.C.V. The characters like radicle length, radicle elongation rate, hypocotyl elongation rate, seedling length, cotyledonary area, seedling fresh weight and specific seedling water content have lesser value of error variance then their respective G.C.V. and P.C.V. in all the environments. These results indicate that the characters in reference though controlled genetically but are greatly affected by environment. (Tables 1 and 2 ) The decrease in seed germination and seedling growth due to heavy metal treatment is in conformity with the findings of other researchers (Ayaz and Kadioglu, 1997; Morzek and Funiceli, 1982; Iqbal and Mehmood, 1991; Jamal et al., 2006). For example, Rahman et al. (2010) observed a reduction in seed germination and seedling growth in chickpea treated with 50,100, 200 and 400 ppm of nickel and cobalt. Singh et al. (2007) observed a reduction in germination percentage and early growth stage of wheat treated with copper at 5, 25, 50, and $100 \mathrm{ppm}$. Treatment of Leucaena leucocephala with 25, 50, 75 and $100 \mathrm{ppm}$ of lead and cadmium showed a gradual reduction in seed germination and seedling growth (Shafiq et al., 2008). However, germination test showed a non significant effect on germination percentage of corn treated with low levels of zinc and copper (6 to $12 \mathrm{ppm}$ ) (Mahmood et al., 2005). According to Shafiq et al. (2008), decrease in seed germination of plant can be attributed to the accelerated breakdown of stored food materials in seed, by the application of heavy metal mixture. Reduction in seed germination can also be attributed to alterations of selection permeability properties of cell membrane. The G.C.V. is lower than P.C.V. for all the characters in all the concentrations (environment). This indicates that these characters are sensitive to environmental influence and the direct selection for these traits on phenotypic basis could be done reliably. The influence of environment of each trait could be determined by the difference in P.C.V. and G.C.V. When the difference between P.C.V. and G.C.V. is less, it suggests that these traits are least affected by 
Table 1. Analysis of variance in different concentrations in Linum usitatissimum L. seed germination.

\begin{tabular}{|c|c|c|c|c|c|c|c|c|}
\hline $\begin{array}{l}\text { Characters/ } \\
\text { treatments/ } \\
\text { observations }\end{array}$ & $\begin{array}{c}\text { Degree } \\
\text { of } \\
\text { freedom } \\
\end{array}$ & $\begin{array}{c}\text { Germination } \\
(\%)\end{array}$ & $\begin{array}{l}\text { Germination } \\
\text { rate index }\end{array}$ & $\begin{array}{l}\text { Radicle } \\
\text { length }\end{array}$ & $\begin{array}{c}\text { Radicle } \\
\text { elongation } \\
\text { rate } \\
\end{array}$ & $\begin{array}{l}\text { Hypocotyl } \\
\text { length }\end{array}$ & $\begin{array}{c}\text { Hypocotyl } \\
\text { elongation } \\
\text { rate } \\
\end{array}$ & $\begin{array}{c}\text { Seedling } \\
\text { length }\end{array}$ \\
\hline \multicolumn{9}{|l|}{ Replication } \\
\hline Unsoaked control & 3 & 2.0833 & 4000.0000 & 0.006693 & 0.002728 & 0.01761 & 0.003636 & 0.04541 \\
\hline Soaked control & 3 & 7.9166 & 15429.333 & 0.01190 & 0.003115 & 0.04117 & 0.01528 & 0.8984 \\
\hline $10^{-5} \mathrm{MCoCl}_{2}$ & 3 & 1.8229 & 1110.6667 & 0.01343 & 0.002993 & 0.04496 & 0.008782 & 0.06563 \\
\hline $10^{-6} \mathrm{M} \mathrm{CoCl}_{2}$ & 3 & 9.3229 & 6533.3333 & 0.02707 & 3263.6894 & 0.02209 & 0.007830 & 0.08374 \\
\hline $10^{-7} \mathrm{M} \mathrm{CoCl}_{2}$ & 3 & 11.2500 & 15717.333 & 0.02394 & 0.007053 & 0.04606 & 0.03235 & 0.1298 \\
\hline $10^{-5} \mathrm{M} \mathrm{HgCl}_{2}$ & 3 & 22.7083 & 7690.6667 & 0.0000 & 0.0000 & 0.0000 & 0.0000 & 0.0000 \\
\hline $10^{-6} \mathrm{M} \mathrm{HgCl}_{2}$ & 3 & 3.0729 & 717.3333 & 0.01700 & 0.002499 & 0.01947 & 0.004961 & 0.06469 \\
\hline $10^{-7} \mathrm{M} \mathrm{HgCl}_{2}$ & 3 & 0.9895 & 2357.3333 & 0.01889 & 0.004299 & 0.006835 & 0.003988 & 0.03580 \\
\hline \multicolumn{9}{|l|}{ Treatment } \\
\hline Unsoaked control & 9 & $124.7222^{\star *}$ & $105756.44^{* *}$ & $1.3309^{* *}$ & $0.06038^{* *}$ & $6.1055^{\star *}$ & $0.1668^{* *}$ & $8.7095^{\star *}$ \\
\hline Soaked control & 9 & $55.5555^{\star \star}$ & $39923.556^{* *}$ & $1.4370^{* *}$ & $0.04888^{* *}$ & $6.2484^{* *}$ & $0.2033^{\star *}$ & $9.2171^{* *}$ \\
\hline $10^{-5} \mathrm{MCoCl}_{2}$ & 9 & $156.0590^{\star \star}$ & $100870.67^{\star *}$ & $1.8494^{\star *}$ & $0.06642^{\star \star}$ & $6.8151^{* *}$ & $0.2387^{\star *}$ & $13.1765^{\star \star}$ \\
\hline $10^{-6} \mathrm{M} \mathrm{CoCl}_{2}$ & 9 & $137.6562^{\star \star}$ & $95688.889^{* *}$ & $1.2758^{\star *}$ & 03272.5959 & $7.2305^{\star *}$ & $0.2071^{* *}$ & $10.6921^{\text {** }}$ \\
\hline $10^{-7} \mathrm{M} \mathrm{CoCl}_{2}$ & 9 & $177.5000^{\star \star}$ & $133985.78^{\star *}$ & $1.1138^{* *}$ & $0.04050^{* *}$ & $5.2634^{* *}$ & $0.1687^{* *}$ & $7.1067^{* *}$ \\
\hline $10^{-5} \mathrm{M} \mathrm{HgCl}_{2}$ & 9 & $179.4444^{\star \star}$ & $103548.898^{* *}$ & 0.0000 & 0.0000 & 0.0000 & 0.0000 & 0.0000 \\
\hline $10^{-6} \mathrm{M} \mathrm{HgCl}_{2}$ & 9 & $139.7395^{\star \star}$ & $86120.889^{\star *}$ & $0.8035^{\star *}$ & $0.02274^{* *}$ & $4.8816^{\star *}$ & $0.1744^{\star *}$ & $7.7607^{\star *}$ \\
\hline $10^{-7} \mathrm{M} \mathrm{HgCl}_{2}$ & 9 & $56.7534^{\star \star}$ & $48608.000^{\star *}$ & $0.8747^{\star *}$ & $0.03043^{\star *}$ & $5.5108^{\star *}$ & $0.2095^{\star *}$ & $5.6365^{\star *}$ \\
\hline \multicolumn{9}{|l|}{ Error } \\
\hline Unsoaked control & 27 & 4.39814 & 4625.7778 & 0.002224 & 0.0006125 & 0.004905 & 0.001072 & 0.006600 \\
\hline Soaked control & 27 & 10.2314 & 5254.5185 & 0.005319 & 0.002000 & 0.008183 & 0.003261 & 0.01312 \\
\hline $10^{-5} \mathrm{MCoCl}_{2}$ & 27 & 5.6423 & 5758.074 & 0.005051 & 0.0008766 & 0.004215 & 0.0007230 & 0.009010 \\
\hline $10^{-6} \mathrm{M} \mathrm{CoCl}_{2}$ & 27 & 5.5806 & 4725.3333 & 0.002814 & 3264.5541 & 0.005221 & 0.002219 & 0.008174 \\
\hline $10^{-7} \mathrm{M} \mathrm{CoCl}_{2}$ & 27 & 4.7685 & 50518519 & 0.001966 & 0.0005661 & 0.007459 & 0.004929 & 0.008210 \\
\hline $10^{-5} \mathrm{M} \mathrm{HgCl}_{2}$ & 27 & 5.2314 & 3257.9259 & 0.0000 & 0.0000 & 0.0000 & 0.0000 & 0.0000 \\
\hline $10^{-6} \mathrm{M} \mathrm{HgCl}_{2}$ & 27 & 9.5543 & 5352.5926 & 0.003142 & 0.0006204 & 0.009150 & 0.001773 & 0.01399 \\
\hline $10^{-7} \mathrm{M} \mathrm{HgCl}_{2}$ & 27 & 7.1238 & 7592.5926 & 0.01034 & 0.001342 & 0.009688 & 0.002753 & 0.01649 \\
\hline
\end{tabular}

${ }^{*}$ Exceed $5 \%$ level of significance; ${ }^{* *}$ exceed $1 \%$ level of significance.

environment. This is also supported by very high value of heritability (Table 2).

High heritability with high/moderate genetic advance has been recorded for all the characters in all the treatment in all the studied varieties. It indicates that expression of these attributes is governed by additive gene effects. High heritability with high genetic advance provides good scope of further improvement by selection. These characters can be subjected to mass progeny, family or any other modified selection scheme for exploiting the additive genetic variance. High heritability estimates have been found to be helpful in making selection of superior genotypes on the basis of phenotypic performance of characters. (Table 2). Plant species shows a wide genetic diversity that offer great sensitivity and selectivity. They have a number of general and specific mechanisms in gene expression that they use to response to unfavorable conditions. These genes linked with a variety of toxic compound response.

The seedling vigour is an important attribute in seed technology. The capacity of seed germination and seedling establishment of variety in a range of environment (climatic condition and heavy metal pollution) has an importance equal to that of its growth and yield potential. Eberhart and Russell (1966) have pointed out that the validity of character's stability increase as the number of environment increases. Hutchinson (1984) and Dueck et al. (1987) are of the opinion that the difference in toxicity level or tolerance is either due to genetic variability or the interactive effect of combination of contamination. Kulkarni and Nayeem (1986) found that in wheat varieties there is genotypic difference in emergence. Gartside and Mc Neily (1974), Bradshaw (1984), Baker and Proctor (1990) and Bradshaw (1991) accepted that only those plant species possessing the required genetic variation can develop 
Table 1. Continued.

\begin{tabular}{|c|c|c|c|c|c|c|c|}
\hline $\begin{array}{l}\text { Characters/ } \\
\text { treatments/ } \\
\text { observations }\end{array}$ & $\begin{array}{c}\text { Cotyledonary } \\
\text { area }\end{array}$ & $\begin{array}{l}\text { Seedling } \\
\text { fresh } \\
\text { weight }\end{array}$ & $\begin{array}{l}\text { Seedling } \\
\text { dry } \\
\text { weight }\end{array}$ & $\begin{array}{c}\text { Absolute } \\
\text { seedling } \\
\text { water } \\
\text { content } \\
\end{array}$ & $\begin{array}{c}\text { Specific } \\
\text { seedling } \\
\text { water } \\
\text { content } \\
\end{array}$ & $\begin{array}{l}\text { Seedling } \\
\text { vigour }\end{array}$ & $\begin{array}{l}\text { Seedling } \\
\text { Vigour } \\
\text { Index }\end{array}$ \\
\hline \multicolumn{8}{|l|}{ Replication } \\
\hline Unsoaked control & 0.0002490 & 10.7229 & 0.4763 & 6.6875 & 7.0162 & 785.3333 & 4229.0000 \\
\hline Soaked control & 0.0005133 & 36.3750 & 0.08567 & 18921.740 & 2.7652 & 2734.666 & 1239.1667 \\
\hline $10^{-5} \mathrm{M} \mathrm{CoCl}_{2}$ & 0.0006492 & 11.0598 & 0.04091 & 9.6497 & 0.4947 & 310.0000 & 168.5833 \\
\hline $10^{-6} \mathrm{M} \mathrm{CoCl}_{2}$ & 0.001020 & 21.2916 & 0.03116 & 19.6822 & 0.1816 & 2662.3333 & 345.9166 \\
\hline $10^{-7} \mathrm{M} \mathrm{CoCl}_{2}$ & 0.0004158 & 6.8020 & 0.1573 & 4.9062 & 2.6240 & 1602.0000 & 1517.0833 \\
\hline $10^{-5} \mathrm{M} \mathrm{HgCl}_{2}$ & 0.0000 & 0.0000 & 0.0000 & 0.0000 & 0.0000 & 0.0000 & 0.0000 \\
\hline $10^{-6} \mathrm{M} \mathrm{HgCl}_{2}$ & 0.0003367 & 5.5963 & 0.04299 & 4.8151 & 0.1083 & 378.8333 & 180.4375 \\
\hline $10^{-7} \mathrm{M} \mathrm{HgCl}_{2}$ & 0.001070 & 0.6822 & 0.03665 & 0.3125 & 2.3561 & 358.6666 & 585.8333 \\
\hline \multicolumn{8}{|l|}{ Treatment } \\
\hline Unsoaked control & $0.01845^{\star \star}$ & $1259.9653^{* *}$ & $2.5149^{\star *}$ & $1153.3472^{* *}$ & $7.1292^{\star *}$ & $99537.7778^{* *}$ & $18016.556^{\star *}$ \\
\hline Soaked control & $0.01113^{\star *}$ & $1278.729^{\star \star}$ & $3.4105^{\star \star}$ & $26843.111^{* *}$ & $5.3937^{\star \star}$ & $104065.33^{\star *}$ & $26290.000^{* *}$ \\
\hline $10^{-5} \mathrm{MCoCl}_{2}$ & $0.01992^{\star \star}$ & $127.6814^{\star \star}$ & $0.5002^{* *}$ & $114.1827^{\star *}$ & $12.9835^{\star \star}$ & $61793.278^{* *}$ & $2801.8472^{* *}$ \\
\hline $10^{-6} \mathrm{M} \mathrm{CoCl}_{2}$ & $0.01407^{\star \star}$ & $438.9765^{\star \star}$ & $1.1405^{\star *}$ & $397.5564^{\star *}$ & $5.7249^{\star *}$ & $79267.889^{* *}$ & $5879.8472^{* *}$ \\
\hline $10^{-7} \mathrm{M} \mathrm{CoCl}_{2}$ & $0.02104^{\star *}$ & $759.0763^{\star \star}$ & $1.7672^{* *}$ & $691.0989^{\star *}$ & $6.9953^{\star *}$ & $82754.000^{* *}$ & $12281.306^{* *}$ \\
\hline $10^{-5} \mathrm{M} \mathrm{HgCl}_{2}$ & 0.0000 & 0.0000 & 0.0000 & 0.0000 & 0.0000 & 0.0000 & 0.0000 \\
\hline $10^{-6} \mathrm{M} \mathrm{HgCl}_{2}$ & $0.02435^{\star *}$ & $468.414^{\star *}$ & $1.6409^{\star *}$ & $420.3237^{\star *}$ & $10.9287^{* *}$ & $47984.167^{* *}$ & $6749.2431^{* *}$ \\
\hline $10^{-7} \mathrm{M} \mathrm{HgCl}_{2}$ & $0.01745^{\star *}$ & $693.7465^{\star \star}$ & $1.4410^{\star *}$ & $634.4930^{\star *}$ & $7.4405^{\star \star}$ & $46266.000^{* *}$ & $8439.5556^{* *}$ \\
\hline \multicolumn{8}{|l|}{ Error } \\
\hline Unsoaked control & 0.0001658 & 1.2546 & 0.04485 & 0.9930 & 0.9180 & 540.8888 & 4037037 \\
\hline Soaked control & 0.0007077 & 13.2141 & 0.03029 & 1.9575 & 0.9189 & 737.3333 & 428.8518 \\
\hline $10^{-5} \mathrm{M} \mathrm{CoCl}_{2}$ & 0.0004250 & 3.1171 & 0.01424 & 2.8123 & 0.5087 & 311.8703 & 77.6944 \\
\hline $10^{-6} \mathrm{M} \mathrm{CoCl}_{2}$ & 0.0002959 & 4.3634 & 0.01435 & 3.9296 & 0.1467 & 439.8888 & 116.1481 \\
\hline $10^{-7} \mathrm{M} \mathrm{CoCl}_{2}$ & 0.0006991 & 1.6932 & 0.03610 & 1.3269 & 0.7584 & 497.0370 & 259.2870 \\
\hline $10^{-5} \mathrm{M} \mathrm{HgCl}_{2}$ & 0.0000 & 0.0000 & 0.0000 & 0.0000 & 0.0000 & 0.0000 & 0.0000 \\
\hline $10^{-6} \mathrm{M} \mathrm{HgCl}_{2}$ & 0.00030700 & 1.1773 & 0.008928 & 1.0318 & 1.9530 & 459.2592 & 122.7754 \\
\hline $10^{-7} \mathrm{M} \mathrm{HgCl}_{2}$ & 0.0003107 & 0.1550 & 0.04611 & 0.1168 & 0.4354 & 961.4814 & 193.5185 \\
\hline
\end{tabular}

tolerance in their population. Bates (1940), suggested that albinism in citrus seedling treated with mercury may be due to primarily to heterozygosis, and perhaps to the presence of unstable genes. In the present study we have used two heavy metals with three concentrations of each and compare it with the control, to obtain the true nature and interaction of heavy metals on seed germination and seedling vigour. The above view is in conformity with those reported by Wilkins (1978) and Coughtrey et al. (1979). Wilkins (1978) stated that ideally a concentration should be used which slightly reduce the growth of tolerant plant. Coughtrey et al. (1979) reported that the use of single metal in tolerance test may not give the reflection of the true nature of interaction between plants and metals. Baker (1987) and Mcnair (1990) found that if a single metal solution is employed over a fixed time period, the data can be highly misleading. Monzuroglu and Geckil (2002) reported the complete inhibition on germination in wheat and cucumber at concentration $>1.5 \mathrm{mM}$ in cucumber and $1.7 \mathrm{mM}$ in wheat.

A perusal of data reveals that the maximum toxic effect and minimum tolerance capacity in $10^{-5} \mathrm{M} \mathrm{CoCl}_{2}$ has been recorded for the characteristics like seed vigour index, seedling vigour and absolute seedling water content. The maximum toxicity and minimum tolerance capacity in 10 ${ }^{6} \mathrm{M} \mathrm{CoCl}_{2}$ has been recorded for the characteristics or variables like seed vigour index, absolute seedling water content and seedling vigour. The $10^{-7} \mathrm{M} \mathrm{CoCl}_{2}$ showed the maximum toxic effect and minimum tolerance for the characters like absolute seedling water content; seed vigour index and seedling dry weight. The minimum toxic effect and maximum tolerance capacity has been observed for the characters like specific seedling water content in all the concentration except in $10^{-7} \mathrm{M} \mathrm{CoCl}_{2}$ where it is observed for character like cotyledonary 
Table 2. Analysis of variability in different concentrations in Linum usitatissimum L. seed germination.

\begin{tabular}{|c|c|c|c|c|c|c|c|c|c|c|c|c|c|c|}
\hline $\begin{array}{l}\text { Characters/ } \\
\text { treatments/ } \\
\text { observations }\end{array}$ & $\begin{array}{c}\text { Germination } \\
(\%)\end{array}$ & $\begin{array}{l}\text { Germination } \\
\text { rate index }\end{array}$ & $\begin{array}{l}\text { Radicle } \\
\text { length }\end{array}$ & $\begin{array}{l}\text { Radicle } \\
\text { elongation } \\
\text { rate }\end{array}$ & $\begin{array}{l}\text { Hypocotyl } \\
\text { length }\end{array}$ & $\begin{array}{l}\text { Hypocotyl } \\
\text { elongation } \\
\text { rate }\end{array}$ & $\begin{array}{l}\text { Seedling } \\
\text { length }\end{array}$ & $\begin{array}{c}\text { Cotyledonary } \\
\text { area }\end{array}$ & $\begin{array}{l}\text { Seedling } \\
\text { fresh } \\
\text { weight }\end{array}$ & $\begin{array}{c}\text { Seedling } \\
\text { dry } \\
\text { weight }\end{array}$ & $\begin{array}{c}\text { Absolute } \\
\text { seedling } \\
\text { water } \\
\text { content }\end{array}$ & $\begin{array}{c}\text { Specific } \\
\text { seedling } \\
\text { water } \\
\text { content }\end{array}$ & $\begin{array}{l}\text { Seedling } \\
\text { vigour }\end{array}$ & $\begin{array}{c}\text { Seedling } \\
\text { vigour } \\
\text { index }\end{array}$ \\
\hline \multicolumn{15}{|l|}{ Mean } \\
\hline Unsoaked control & 89.75 & 2087.80 & 4.12 & 0.76 & 7.11 & 1.30 & 11.24 & 0.43 & 84.98 & 4.02 & 80.96 & 20.12 & 1012.66 & 360.58 \\
\hline Soaked control & 93.75 & 2175.37 & 3.93 & 0.71 & 7.25 & 1.32 & 11.19 & 0.43 & 83.87 & 4.61 & 101.75 & 17.10 & 1053.60 & 433.79 \\
\hline $10^{-5} \mathrm{M} \mathrm{CoCl}_{2}$ & 58.81 & 1291.70 & 2.35 & 0.44 & 4.10 & 0.71 & 6.45 & 0.31 & 41.28 & 2.12 & 39.16 & 18.50 & 383.12 & 126.14 \\
\hline $10^{-6} \mathrm{M} \mathrm{CoCl}_{2}$ & 68.68 & 1528.62 & 3.00 & 0.54 & 5.53 & 1.01 & 8.53 & 0.35 & 54.43 & 2.82 & 51.61 & 18.25 & 592.66 & 194.19 \\
\hline $10^{-7} \mathrm{M} \mathrm{CoCl}_{2}$ & 81.00 & 1842.57 & 3.63 & 0.66 & 6.77 & 1.19 & 10.41 & 0.41 & 72.16 & 3.64 & 68.52 & 18.78 & 846.68 & 295.44 \\
\hline $10^{-5} \mathrm{M} \mathrm{HgCl}_{2}$ & 45.12 & 962.90 & 0.00 & 0.00 & 0.00 & 0.00 & 0.00 & 0.00 & 0.00 & 0.00 & 0.00 & 0.00 & 0.00 & 0.00 \\
\hline $10^{-6} \mathrm{M} \mathrm{HgCl}_{2}$ & 59.31 & 1309.10 & 2.62 & 0.47 & 4.46 & 0.79 & 7.08 & 0.30 & 51.99 & 2.64 & 49.35 & 18.87 & 425.07 & 158.08 \\
\hline $10^{-7} \mathrm{M} \mathrm{HgCl}_{2}$ & 71.93 & 1606.77 & 3.40 & 0.62 & 6.46 & 1.20 & 9.87 & 0.38 & 69.47 & 3.47 & 66.00 & 19.02 & 711.27 & 249.57 \\
\hline \multicolumn{15}{|l|}{ G.C.V } \\
\hline Unsoaked control & 6.1 & 7.62 & 13.97 & 15.97 & 17.36 & 15.61 & 13.12 & 15.50 & 20.87 & 19.52 & 20.96 & 6.19 & 15.54 & 18.40 \\
\hline Soaked control & 3.59 & 4.28 & 5.21 & 15.17 & 17.21 & 16.86 & 13.55 & 11.68 & 21.21 & 19.92 & 41.89 & 6.18 & 15.25 & 18.54 \\
\hline $10^{-5} \mathrm{M} \mathrm{CoCl}_{2}$ & 10.43 & 11.94 & 28.82 & 28.57 & 31.82 & 34.28 & 28.10 & 22.25 & 13.52 & 16.42 & 13.47 & 9.54 & 32.36 & 20.69 \\
\hline $10^{-6} \mathrm{M} \mathrm{CoCl}_{2}$ & 8.36 & 9.87 & 18.75 & 14.81 & 24.30 & 22.34 & 19.14 & 16.58 & 19.15 & 18.77 & 19.22 & 6.47 & 23.69 & 19.55 \\
\hline $10^{-7} \mathrm{M} \mathrm{CoCl}_{2}$ & 8.11 & 9.74 & 14.51 & 15.10 & 16.91 & 16.99 & 12.79 & 17.07 & 19.07 & 18.03 & 19.16 & 6.65 & 16.94 & 18.56 \\
\hline $10^{-5} \mathrm{M} \mathrm{HgCl}_{2}$ & 14.62 & 16.44 & 0.00 & 0.00 & 0.00 & 0.00 & 0.00 & 0.00 & 0.00 & 0.00 & 0.00 & 0.00 & 0.00 & 0.00 \\
\hline $10^{-6} \mathrm{M} \mathrm{HgCl}_{2}$ & 9.62 & 10.85 & 17.06 & 15.79 & 24.72 & 26.19 & 19.64 & 25.30 & 20.79 & 24.15 & 20.74 & 7.93 & 25.64 & 25.75 \\
\hline $10^{-7} \mathrm{M} \mathrm{HgCl}_{2}$ & 4.90 & 6.30 & 13.66 & 13.69 & 18.13 & 18.81 & 13.03 & 16.85 & 18.95 & 17.02 & 19.08 & 6.96 & 14.96 & 18.19 \\
\hline \multicolumn{15}{|l|}{ P.C.V } \\
\hline Unsoaked control & 6.54 & 8.28 & 14.02 & 16.30 & 17.39 & 15.81 & 13.14 & 15.78 & 20.91 & 20.22 & 21.00 & 7.81 & 15.70 & 19.23 \\
\hline Soaked control & 4.95 & 5.42 & 15.32 & 16.41 & 17.25 & 17.40 & 13.59 & 13.17 & 21.64 & 20.27 & 143.75 & 8.35 & 15.47 & 19.14 \\
\hline $10^{-5} \mathrm{M} \mathrm{CoCl}_{2}$ & 11.18 & 13.31 & 28.98 & 29.33 & 31.86 & 34.48 & 28.14 & 23.20 & 14.18 & 17.36 & 14.14 & 10.29 & 32.69 & 21.48 \\
\hline $10^{-6} \mathrm{M} \mathrm{CoCl}_{2}$ & 9.07 & 10.84 & 18.84 & 16.81 & 24.34 & 22.82 & 19.17 & 17.28 & 19.53 & 19.24 & 19.60 & 6.80 & 23.95 & 20.32 \\
\hline $10^{-7} \mathrm{M} \mathrm{CoCl}_{2}$ & 8.55 & 10.48 & 14.56 & 15.52 & 16.96 & 17.99 & 12.84 & 18.21 & 19.15 & 18.77 & 19.24 & 8.10 & 17.14 & 19.34 \\
\hline $10^{-5} \mathrm{M} \mathrm{HgCl}_{2}$ & 15.48 & 17.48 & 0.00 & 0.00 & 0.00 & 0.00 & 0.00 & 0.00 & 0.00 & 0.00 & 0.00 & 0.00 & 0.00 & 0.00 \\
\hline $10^{-6} \mathrm{M} \mathrm{HgCl}_{2}$ & 10.49 & 12.21 & 17.19 & 16.65 & 24.81 & 26.73 & 19.71 & 25.94 & 20.89 & 24.41 & 20.85 & 10.85 & 26.13 & 26.68 \\
\hline $10^{-7} \mathrm{M} \mathrm{HgCl}_{2}$ & 6.14 & 8.31 & 13.98 & 14.90 & 18.19 & 19.30 & 13.10 & 17.45 & 18.96 & 18.11 & 19.09 & 7.77 & 15.58 & 19.03 \\
\hline
\end{tabular}

area. These results indicate that in cobalt the $10^{-}$ ${ }^{5} \mathrm{M}$ concentration is much toxic than $10^{-6} \mathrm{M}$ concentration and $10^{-7} \mathrm{M} \mathrm{CoCl}_{2}$ concentration seem to be normal, but it shows toxicity to some extent. The same toxicity level shows difference for the characters in different concentration. It could be due to the disrupting capacity of the applied concentration of metal to disrupt the integrity of biomolecules of the 
Table 2. Continued.

\begin{tabular}{|c|c|c|c|c|c|c|c|c|c|c|c|c|c|c|}
\hline \multicolumn{15}{|l|}{ Heritability } \\
\hline Unsoaked control & 0.872 & 0.845 & 0.993 & 0.961 & 0.987 & 0.975 & 0.967 & 0.965 & 0.986 & 0.932 & 0.977 & 0.628 & 0.979 & 0.916 \\
\hline Soaked control & 0.525 & 0.623 & 0.985 & 0.854 & 0.985 & 0.939 & 0.974 & 0.786 & 0.960 & 0.965 & 0.985 & 0.549 & 0.972 & 0.938 \\
\hline $10^{-5} \mathrm{M} \mathrm{CoCl}_{2}$ & 0.870 & 0.805 & 0.989 & 0.949 & 0.988 & 0.988 & 0.977 & 0.920 & 0.909 & 0.895 & 0.908 & 0.807 & 0.980 & 0.898 \\
\hline $10^{-6} \mathrm{M} \mathrm{CoCl}_{2}$ & 0.849 & 0.828 & 0.981 & 0.962 & 0.987 & 0.958 & 0.967 & 0.921 & 0.961 & 0.951 & 0.962 & 0.905 & 0.978 & 0.925 \\
\hline $10^{-7} \mathrm{M} \mathrm{CoCl}_{2}$ & 0.901 & 0.865 & 0.993 & 0.946 & 0.984 & 0.893 & 0.985 & 0.879 & 0.981 & 0.923 & 0.992 & 0.673 & 0.976 & 0.921 \\
\hline $10^{-5} \mathrm{M} \mathrm{HgCl}_{2}$ & 0.893 & 0.885 & 0.00 & 0.00 & 0.00 & 0.00 & 0.00 & 0.00 & 0.00 & 0.00 & 0.00 & 0.00 & 0.00 & 0.00 \\
\hline $10^{-6} \mathrm{M} \mathrm{HgCl}_{2}$ & 0.773 & 0.790 & 0.985 & 0.899 & 0.993 & 0.961 & 0.993 & 0.991 & 0.951 & 0.979 & 0.990 & 0.535 & 0.963 & 0.931 \\
\hline $10^{-7} \mathrm{M} \mathrm{HgCl}_{2}$ & 0.635 & 0.575 & 0.954 & 0.844 & 0.993 & 0.994 & 0.990 & 0.932 & 0.986 & 0.883 & 0.973 & 0.801 & 0.922 & 0.914 \\
\hline \multicolumn{15}{|l|}{ Genetic advance } \\
\hline Unsoaked control & 10.55 & 301.16 & 1.18 & 0.25 & 2.54 & 0.41 & 3.03 & 0.14 & 36.47 & 1.56 & 34.90 & 2.04 & 320.59 & 13.83 \\
\hline Soaked Control & 5.03 & 151.32 & 1.22 & 0.21 & 2.57 & 0.45 & 3.12 & 0.09 & 35.90 & 1.86 & 25.59 & 1.61 & 326.46 & 160.40 \\
\hline $10^{-5} \mathrm{M} \mathrm{CoCl}_{2}$ & 11.78 & 285.02 & 1.39 & 0.26 & 2.68 & 0.50 & 3.73 & 0.14 & 10.96 & 0.68 & 10.36 & 3.37 & 252.84 & 50.93 \\
\hline $10^{-6} \mathrm{M} \mathrm{CoCl}_{2}$ & 10.90 & 282.67 & 1.16 & 0.27 & 2.76 & 0.46 & 3.36 & 0.12 & 21.05 & 1.07 & 20.04 & 2.31 & 286.01 & 75.22 \\
\hline $10^{-7} \mathrm{M} \mathrm{CoCl}_{2}$ & 12.85 & 343.88 & 1.08 & 0.20 & 2.35 & 0.39 & 2.74 & 0.14 & 28.22 & 1.30 & 26.95 & 2.11 & 291.90 & 108.36 \\
\hline $10^{-5} \mathrm{M} \mathrm{HgCl}_{2}$ & 12.85 & 306.86 & 0.00 & 0.00 & 0.00 & 0.00 & 0.00 & 0.00 & 0.00 & 0.00 & 0.00 & 0.00 & 0.00 & 0.00 \\
\hline $10^{-6} \mathrm{M} \mathrm{HgCl}_{2}$ & 10.33 & 260.25 & 0.91 & 0.15 & 2.27 & 0.42 & 2.66 & 0.16 & 22.15 & 1.30 & 20.99 & 2.26 & 220.32 & 80.90 \\
\hline $10^{-7} \mathrm{M} \mathrm{HgCl}_{2}$ & 5.78 & 158.12 & 0.94 & 0.16 & 2.41 & 0.46 & 2.64 & 0.13 & 27.41 & 1.14 & 25.93 & 2.44 & 210.48 & 89.43 \\
\hline \multicolumn{15}{|l|}{$\begin{array}{l}\text { G.A in \% mean } \\
(\mathrm{K}=2.06)\end{array}$} \\
\hline Unsoaked control & 11.75 & 14.12 & 28.64 & 32.89 & 35.72 & 31.53 & 26.95 & 32.35 & 42.91 & 38.80 & 43.10 & 10.13 & 31.65 & 36.28 \\
\hline Soaked control & 5.36 & 6.95 & 31.04 & 29.57 & 35.44 & 34.09 & 27.88 & 20.93 & 42.80 & 40.34 & 25.14 & 9.41 & 30.98 & 36.97 \\
\hline $10^{-5} \mathrm{M} \mathrm{CoCl}_{2}$ & 20.03 & 22.06 & 59.14 & 59.09 & 65.36 & 70.42 & 57.82 & 45.16 & 26.55 & 32.07 & 26.45 & 18.21 & 65.99 & 40.37 \\
\hline $10^{-6} \mathrm{M} \mathrm{CoCl}_{2}$ & 15.87 & 18.49 & 38.66 & 50.00 & 49.90 & 45.54 & 39.39 & 34.28 & 38.67 & 37.94 & 38.82 & 12.48 & 48.25 & 38.73 \\
\hline $10^{-7} \mathrm{M} \mathrm{CoCl}_{2}$ & 15.86 & 18.66 & 29.75 & 30.30 & 34.71 & 32.77 & 26.32 & 34.14 & 39.10 & 35.71 & 39.33 & 11.23 & 34.47 & 36.67 \\
\hline $10^{-5} \mathrm{M} \mathrm{HgCl}_{2}$ & 28.17 & 31.86 & 0.00 & 0.00 & 0.00 & 0.00 & 0.00 & 0.00 & 0.00 & 0.00 & 0.00 & 0.00 & 0.00 & 0.00 \\
\hline $10^{-6} \mathrm{M} \mathrm{HgCl}_{2}$ & 17.41 & 19.88 & 34.73 & 31.94 & 50.89 & 53.16 & 40.39 & 53.33 & 42.60 & 49.24 & 42.53 & 11.97 & 51.83 & 51.17 \\
\hline $10^{-7} \mathrm{M} \mathrm{HgCl}_{2}$ & 8.03 & 9.84 & 27.64 & 25.80 & 37.30 & 38.33 & 26.74 & 34.21 & 39.02 & 32.85 & 39.28 & 12.82 & 29.59 & 35.83 \\
\hline
\end{tabular}

particular region or character. The difference in toxicity level or tolerance capacity is either due to genetic variability or the interaction of contamination. The present findings on cobalt toxicity effect are in agreement to those reported by Haselhoff (1985). Brenchley (1938) and Vergano and Hunter (1952). Haselhoff (1895) reported that $1 \mathrm{ppm}$ cobalt in culture solution was toxic to beans and corns, while Vergano and Hunter (1952) reported that solution culture containing as low as $0.1 \mathrm{ppm}$ cobalt produce adverse or toxic effect on many crop plant.

Blaylock et al. (1986) observed that cobalt generally decreased the photosynthesis and chlorophyll in soybean and tomatoes. Recently, $\mathrm{Xu}$ et al. (1993) reported that Co at low concentration allowed continued growth cycle of groundnut cells $(>0.5 \mathrm{mM})$. At high concentration $(1 \mathrm{mM})$ these ions inhibit the growth cycle of cell. We considered that $1 \times 10^{-4} \mathrm{M} \mathrm{CoCl}_{2}$ concentration is the maximum toxicity and there after no 
germination is possible. Mitchell (1945) considered the $0.7 \mathrm{ppm}$ ratio of cobalt content in plant is the minimum tolerance limit. Chatterjee and Chatterjee (2000) also found similar result in cauliflower. Palit et al. (2008) reported that cobalt act as pre-prophase poison and thus retarded the process of karyokinesis and cytokinesis. The distribution of cobalt in plant is entirely species dependent.

When the seeds of Linum allowed to germinate in $10^{-}$ ${ }^{5} \mathrm{M} \mathrm{HgCl}_{2}$, it showed highest toxic effect. Here only seed germinated and no further seedling establishment was noticed. In this concentration the seeds have loose their tolerance capacity. The $10^{-6} \mathrm{HgCl}_{2}$ Concentration shows less toxic effect in comparison to $10^{-5} \mathrm{M}$. In this concentration, the maximum toxic effect and minimum tolerance capacity has been observed for the followings variables: seedling vigour, absolute seedling water content, seedling dry weight and seed vigour index. However, in $10^{-7} \mathrm{M}$ concentration of $\mathrm{HgCl}_{2}$, the maximum toxic effect has been observed for the seedling vigour, seed vigour index and absolute seedling water content. The minimum toxic effect and maximum tolerance capacity has been observed for the specific seedling water content in both the concentration of $\mathrm{HgCl}_{2}$ in different varieties. These findings also suggest the last limit that is with maximum toxic effect of mercury for these plant species is $10^{-5} \mathrm{M} \mathrm{HgCl}_{2}$ concentration. The $10^{-}$ ${ }^{7} \mathrm{M} \mathrm{HgCl}_{2}$ concentration has least toxic effect (Table 3 ).

The above findings are more or less similar to those reported by Mukherji and Ganguly (1974). They stated that root growth inhibition was stronger than shoot growth inhibition by $\mathrm{Hg}$ treatment. Sharma (1983) soaked the seeds of Pisum sativum cultivars namely $\mathrm{T}-163$, Bonneville and Arkel for $24 \mathrm{~h}$ in $4.98 \times 10^{-5}$ and $2.49 \times 10^{-4} \mathrm{M}$ concentration of mercuric acetate. He observed that in $2.49 \times 10^{-4}$ concentration the germination and growth was inhibited. Sharma (1984) reported that in Cucumis utilissimus and Luffa aegyptiaca on allocation of lower concentration of mercury showed that hypocotyl growth was more affected that of radicle. Jamal et al. (2006) observed the highest reduction was in root length rather than shoot and seedling length due to mercury concentration $<0.05$ in two wheat varieties.

Generally, it makes negative effect on their metabolisms by influencing the activity of cellular enzymes (Yang et al., 1986). Many studies have been carried out on the effects of heavy metals on plants. They showed that, cadmium in certain amounts inhibited the germination and development of the plants (Aydinalp and Marinova, 2009). Cadmium caused chlorophyll aberrations at very high concentrations (Reddy and Vaidyanath, 1978), reduction of mitotic index in root cells (Zhang and Yang, 1994), chromosomal abnormalities and micronucleus formation ( $\mathrm{Li}$ and Zheng, 1992), disorder in nucleus structure (Jiang et al., 1994) and abnormalities in (deoxyribonucleic acid) DNA and Ribonucleic acid (RNA) synthesis (Enger et al., 1997). Several authors reported that, the inhibition of root elongation caused by heavy metals may be due to metal interference with cell division, including inducement of chromosomal aberrations and abnormal mitosis (Jiang et al., 2001; Huillier et al., 1996; Radha et al., 2010; Liu et al., 2003), which can be effected on seedling growth and explain the inhabitation of seedling growth in this investigation. It was also observed that mercury toxicity was more than cobalt and the higher concentration showed more toxic effect than the lower. The present study further indicates that there are limits to the degree of tolerance that plants can achieve. The quantitative limits of tolerance and toxicity have great importance for determining the actual dosage at which a chemical becomes toxic as also the point beyond which no further adaptation can be achieved by a plant species or variety. It is inferred that the evaluation of germination and seedling growth may be helpful in assessing the relative resistance or susceptibility to heavy metal. So the choice of resistant cultivars for cultivation would be meaningful in minimizing the pollution hazards. The possible reason of inhibition of seedling growth due to mercury and cobalt may be to its association with cell wall or cell membrane inhibiting water absorption and interference with mobilization of reserve food from residual cotyledons to the developing seedling. The reason for low tolerance against heavy metal mixtures might be due to changes in the physiological mechanism in seed germination and seedling growth of safflower. Shafiq and Iqbal (2005) reported similar results for low tolerance in Cassia siamea seedlings at $100 \mathrm{ppm}$ of lead and cadmium treatments as compared to control. General observation in this study can conclude that, heavy metal mixture treatment produced toxic impact on germination and seedling growth. Increase in the concentrations of heavy metals mixture in the soil, brought up changes in most of the growth parameters of crop.

Contamination of wastewater with high concentration of heavy metals caused a significant decrease in the number of survivals .The toxicity of heavy metals on organism depends mainly upon two factors, namely, metal species and concentration. Other factors such as $\mathrm{pH}$, influent strength are also reported to affect the toxicity of metals, though to a lesser degree. Heavy metals change the structure of the flora and fauna by modifying both cell density and species richness. Due to the toxic behavior of metal ions resulting in a lower demand of dissolved oxygen. Metal ion complexion with heavy metal effects the growth of living being and it's the cause of death. When a critical amount of heavy metal is applied to the seeds or seedlings either the cell's metabolic activity decreases or the cells die. It could be possible that there is a specific gene which monitors the toxic level.

\section{Correlation coefficient}

As revealed from Table 4, the highly significant positive 
Table 3. Tolerance index and toxicity level in linum usitatissimum seed germination.

\begin{tabular}{|c|c|c|c|c|c|c|c|c|c|c|c|c|c|}
\hline \multirow[b]{2}{*}{$S / N$} & \multirow{2}{*}{$\begin{array}{l}\text { Treatment / } \\
\text { characters }\end{array}$} & \multicolumn{2}{|c|}{$10^{-5} \mathrm{CoCl}_{2}$} & \multicolumn{2}{|c|}{$10^{-6} \mathrm{CoCl}_{2}$} & \multicolumn{2}{|c|}{$10^{-7} \mathrm{CoCl}_{2}$} & \multicolumn{2}{|c|}{$10^{-5} \mathrm{HgCl}_{2}$} & \multicolumn{2}{|c|}{$10^{-6} \mathrm{HgCl}_{2}$} & \multicolumn{2}{|c|}{$10^{-7} \mathrm{HgCl}_{2}$} \\
\hline & & $\begin{array}{l}\text { T.I } \\
(\%)\end{array}$ & T.L (\%) & $\begin{array}{l}\text { T.I } \\
(\%)\end{array}$ & $\begin{array}{l}\text { T.L } \\
(\%)\end{array}$ & $\begin{array}{l}\text { T.I } \\
(\%)\end{array}$ & $\begin{array}{l}\text { T.L } \\
(\%)\end{array}$ & $\begin{array}{l}\text { T.I } \\
(\%) \\
\end{array}$ & $\begin{array}{l}\text { T.L } \\
(\%) \\
\end{array}$ & $\begin{array}{l}\text { T.I } \\
(\%)\end{array}$ & $\begin{array}{l}\text { T.L } \\
(\%) \\
\end{array}$ & $\begin{array}{l}\text { T.I } \\
(\%) \\
\end{array}$ & $\begin{array}{l}\text { T.L } \\
(\%)\end{array}$ \\
\hline 1 & $\%$ Germination & 62.67 & 37.27 & 73.26 & 26.75 & 86.40 & 13.60 & 48.12 & 51.88 & 63.26 & 36.74 & 76.72 & 23.28 \\
\hline 2 & $\begin{array}{l}\text { Germination } \\
\text { rate index }\end{array}$ & 59.37 & 40.63 & 70.26 & 29.74 & 84.70 & 15.30 & 44.26 & 55.74 & 60.17 & 39.83 & 73.86 & 26.14 \\
\hline 3 & Radicle length & 59.79 & 40.21 & 76.33 & 23.67 & 92.36 & 7.46 & 0.00 & 0.00 & 66.66 & 33.34 & 86.51 & 13.49 \\
\hline 4 & $\begin{array}{l}\text { Radicle } \\
\text { elongation } \\
\text { rate }\end{array}$ & 61.97 & 38.03 & 70.05 & 29.95 & 92.95 & 7.05 & 0.00 & 0.00 & 66.19 & 33.81 & 87.32 & 12.68 \\
\hline 5 & $\begin{array}{l}\text { Hypocotyls } \\
\text { length }\end{array}$ & 56.55 & 43.45 & 76.27 & 23.73 & 93.37 & 6.63 & 0.00 & 0.00 & 61.51 & 38.49 & 89.10 & 10.90 \\
\hline 6 & $\begin{array}{l}\text { Hypocotyl } \\
\text { elongation } \\
\text { rate }\end{array}$ & 53.78 & 46.22 & 76.15 & 23.85 & 90.15 & 9.85 & 0.00 & 0.00 & 59.84 & 40.16 & 90.09 & 9.91 \\
\hline 7 & $\begin{array}{l}\text { Seedling } \\
\text { length }\end{array}$ & 57.64 & 42.36 & 76.22 & 23.78 & 93.02 & 6.98 & 0.00 & 0.00 & 63.27 & 36.73 & 88.20 & 11.80 \\
\hline 8 & $\begin{array}{l}\text { Cotyledonary } \\
\text { area }\end{array}$ & 72.09 & 27.91 & 81.39 & 18.61 & 95.34 & 4.66 & 0.00 & 0.00 & 69.76 & 30.24 & 88.37 & 11.63 \\
\hline 9 & $\begin{array}{l}\text { Seedling fresh } \\
\text { weight }\end{array}$ & 49.21 & 50.79 & 64.89 & 35.11 & 86.03 & 13.97 & 0.00 & 0.00 & 61.98 & 38.02 & 76.86 & 23.14 \\
\hline 10 & $\begin{array}{l}\text { Seedling dry } \\
\text { weight }\end{array}$ & 45.98 & 54.02 & 61.17 & 38.83 & 78.95 & 21.05 & 0.00 & 0.00 & 57.26 & 42.74 & 75.27 & 24.73 \\
\hline 11 & $\begin{array}{l}\text { Absolute } \\
\text { seedling } \\
\text { water content }\end{array}$ & 38.48 & 61.52 & 50.72 & 49.28 & 67.34 & 32.66 & 0.00 & 0.00 & 48.50 & 51.50 & 64.86 & 35.14 \\
\hline 12 & $\begin{array}{l}\text { Specific } \\
\text { seedling } \\
\text { water content }\end{array}$ & 90.70 & 9.30 & 91.94 & 8.06 & 93.33 & 6.67 & 0.00 & 0.00 & 93.78 & 6.22 & 94.53 & 5.47 \\
\hline 13 & $\begin{array}{l}\text { Seedling } \\
\text { vigour }\end{array}$ & 36.36 & 63.64 & 56.25 & 43.75 & 80.36 & 19.64 & 0.00 & 0.00 & 40.34 & 59.66 & 67.50 & 57.53 \\
\hline 14 & $\begin{array}{l}\text { Seed vigour } \\
\text { index }\end{array}$ & 29.07 & 70.93 & 44.76 & 55.24 & 68.10 & 31.90 & 0.00 & 0.00 & 36.44 & 40.34 & 57.53 & 42.47 \\
\hline
\end{tabular}

correlation coefficient has been observed among the 1000 seed weight with cotyledonary area absolute seedling water content and seed size, the seed vigour index with radicle length, radicle elongation rate, seedling length, hypocotyl elongation rate, seedling fresh weight, seedling dry weight, absolute seedling water content and seedling vigour, the seedling vigour with radicle length, radicle elongation rate, hypocotyl elongation rate and seedling length, the absolute seedling water content with seedling length, seedling fresh weight and seedling dry weight. The seedling dry weight with seedling fresh weight, the seedling fresh weight with seedling length, the seedling length with hypocotyl length and hypocotyl elongation rate, the hypocotyl elongation rate with hypocotyl length, the radicle elongation rate with radicle length. 
Table 4. Estimation of correlation coefficient Among various characters of seed germination in Linum usitatissimum.

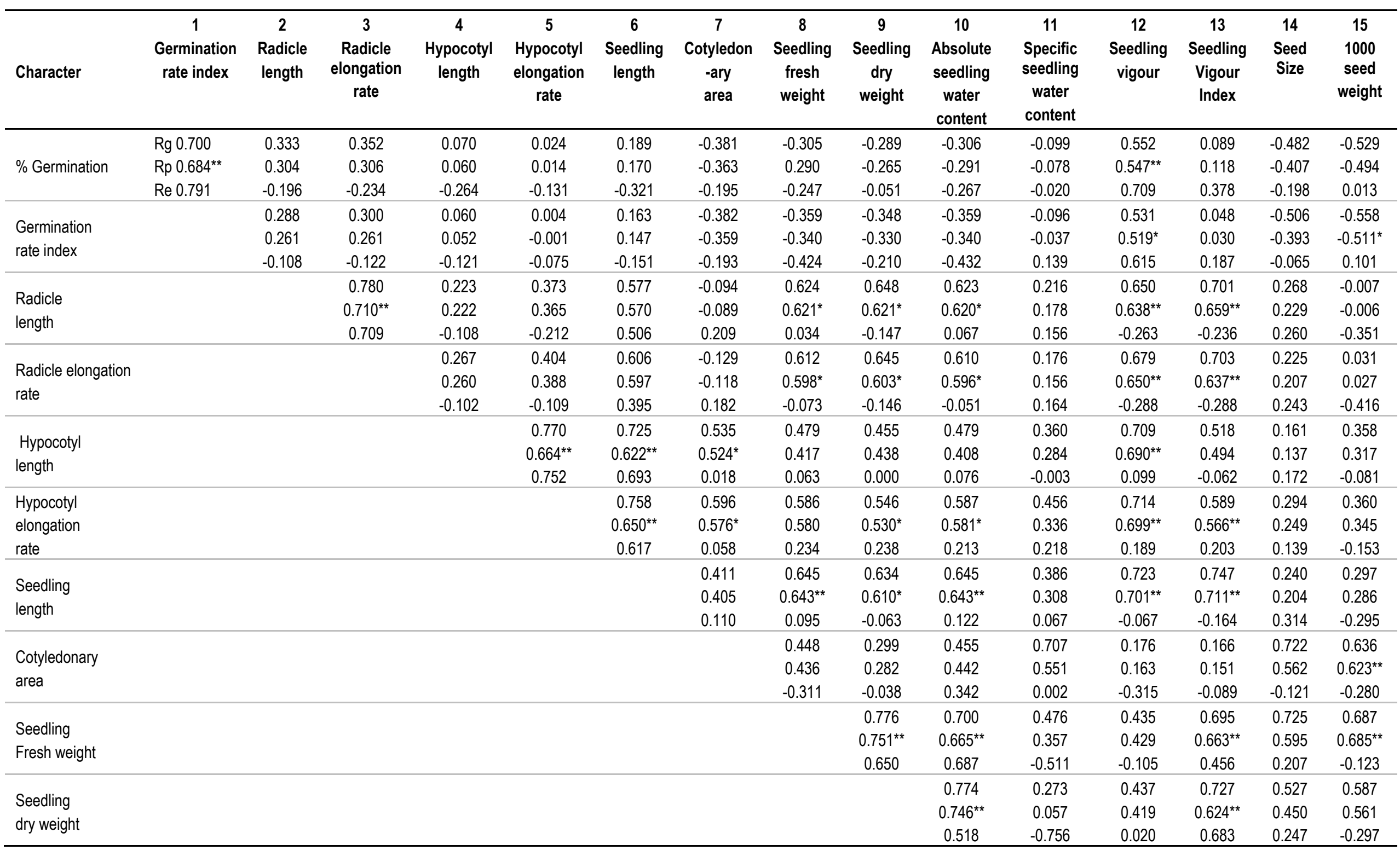


Table 4. Continued.

\begin{tabular}{|c|c|c|c|c|c|}
\hline Absolute & 0.485 & 0.434 & 0.592 & 0.733 & 0.691 \\
\hline seedling & 0.370 & 0.428 & $0.658^{* *}$ & 0.601 & $0.680^{* *}$ \\
\hline $\begin{array}{l}\text { water } \\
\text { content }\end{array}$ & -0.370 & -0.123 & 0.326 & 0.180 & -0.079 \\
\hline $\begin{array}{l}\text { Specific } \\
\text { seedling }\end{array}$ & & 0.273 & 0.245 & 0.693 & 0.603 \\
\hline water & & 0.209 & 0.228 & 0.554 & 0.485 \\
\hline content & & -0.058 & -0.796 & -0.240 & 0.335 \\
\hline Seedling & & & 0.682 & 0.005 & 0.020 \\
\hline vigour & & & 0.660 & -0.004 & 0.020 \\
\hline & & & 0.256 & -0.093 & 0.067 \\
\hline Seedling & & & & 0.352 & 0.400 \\
\hline vigour & & & & 0.299 & 0.380 \\
\hline index & & & & 0.147 & -0.274 \\
\hline & & & & & 0.735 \\
\hline $\begin{array}{l}\text { seed } \\
\text { size }\end{array}$ & & & & & $0.674^{* *}$ \\
\hline & & & & & -0.190 \\
\hline
\end{tabular}

*Significant at $5 \%$ level;**Significant at $1 \%$ level.

The significant positive correlation coefficient has been observed among the 1000 seed weight with seedling dry weight, the seed size with cotyledonary area, seedling fresh weight, absolute seedling water content and specific seedling water content. The seedling vigour with seed germination and germination rate index, the absolute water content with radicle length, radical elongation rate and hypocotyl elongation rate, the seedling dry weight with radicle length, radicle elongation rate and hypocotyl elongation rate, the seedling fresh weight with radicle length, radicle elongation rate and hypocotyl elongation rate, the cotyledonary area with hypocotyl length and hypocotyl elongation rate, the seedling length with radicle length and radicle elongation rate.

The significant negative correlation coefficient has observed between 1000 seed weight with germination rate index.
These findings are similar to those of reported by Carleton and Cooper (1972). They found the positive correlation between seed size and seedling vigour in three forage legume and same was observed by Gelmond (1972) in cotton.

Mulett and Wilkinson (1979) reported that hypocotyl length had significant positive correlation with fresh and dry weight of seedling in Pisum sativum. Hussaini et al. (1984) stated that seed size positively affect the seed germination and vigour in maize.

Nayeem and Deshpande (1987) reported that in wheat, the seed vigour index had significantly positive correlation with radicle length, seedling fresh and dry weight. Eduardo et al. (2007) observed the positive correlation in growth, water content and chlorophyll concentration in Rumex induratus and Marrubium vulgare.

The above discussed results clearly indicate that the genotypic correlation coefficient has found to be greater, than phenotypic and environmental correlation in almost all the characters. This indicates that though these have a high degree of association between two traits at genotypic level, its phenotypic expression has lessened due to the influence of the environment. The negative correlation might arise in character is favoured over the other in the developing stage, when the nutrient supply is limited. In other words this is a case of physiological incompatibility which leads to conclusion that intensification of one such character will be at the expense of other. Possible genetic reason for negative correlation among these components could be pleiotropy.

\section{Path coefficient analysis}

The results of Table 5 indicated that the seedling 
length exerted maximum positive direct effect on seedling vigour followed by characters like absolute seedling water content, percentage germination, seed vigour index and specific seedling water content at phenotypic level. However, seedling fresh weight showed maximum direct effect followed by seedling length, seed vigour index, radicle elongation rate, specific seedling water content, germination rate index and cotyledonary area at genotypic level.

The hypocotyl elongation rate has maximum positive indirect effect on seedling vigour via characteristics seedling length followed by hypocotyl length, seed vigour index, seedling fresh weight, absolute seedling water content, seedling dry weight, radicle elongation rate, radicle length, cotyledonary area, specific seedling water content and 1000 seed weight. The seedling dry weight exert maximum positive indirect effect on seedling vigour via seed vigour index followed by seedling fresh weight, absolute seedling water content, radicle length, radicle elongation rate, seedling length, hypocotyl elongation rate and hypocotyl length. These findings suggest that seedling vigour is a complex character which is governed by many parameters and is an important attribute in seed technology. The direct and indirect effects of characters help in designing appropriate selection strategies and pinpoint the actual parameters to be manipulated. The high positive direct effect which was reflected in its positive and significant correlation coefficient may be regarded as the prime character for selection. This seems to be effective in obtaining superior seedling vigour. However, the characters which have positive and significant correlation coefficient with seedling vigour, but the direct effect was negligible or negative, the indirect effect seem to be cause of positive correlation, therefore, for improving the seedling vigour, the indirect causal factors are to be considered simultaneously for selection. This correlation coefficient has been found to be almost equal to its indirect effect.

The hypocotyl length has maximum negative direct effect on seedling vigour followed by radicle length. These negative direct effects indicate the difficulty to improve the seedling vigour through selection, but compensated through the positive indirect effect via other characters which ultimately resulted in the significant positive correlation. Thus these characters in a balanced proportion and are mainly responsible for the improvement in seedling vigour. The maximum positive direct effect of seedling length on the seedling vigour has been nullified by the negative indirect effect via hypocotyl and radicle length. To overcome this problem a special technique should be adopted to break this linkage. It is interesting to note that the direct and indirect effect have positive value at phenotypic level and negative value at genotypic level or vice versa. It shows that the involvement of these characters in seedling vigour are variable, and the time of improvement if the due weight age would be given to these characters, thus their direction must be considered separately at genotypic and phenotypic level. The residual effect has negative value at genotypic level and positive value at phenotypic level with considerably low magnitude which indicates that most of the important attributed characters enhancing seedling vigour ratio have been taken into account in the study. Basak et al. (2001) observed that heavy metal stressed the chlorophyll content along with Hill activity. Although, the effects of the individual heavy metals on plants have been evaluated by many studies (Brown and Wilkins, 1986; Shafig et al., 2008; Dharam et al., 2007; Shafiq and lqbal, 2005; Kabir et al., 2008), limited information is available on the effects of heavy metal mixture on plant species. There is the need to study the combined effects of heavy metals on plants because most of them are present in an environment at the same time or on the same environment at different times. Linseed is economically an important annual oilseed crop. It has been traditionally grown for its oil, food, fabrics animal meal, bird feed, medicinal uses, as a potential candidate crop for production of plant made pharmaceuticals. To our knowledge, limited information is available on the effects of heavy metals on seed germination and seedling growth of this plant. Hence, the objective of this study was to evaluate the various effects of mixed heavy metals at different levels on Linum in early growth stages.

\section{Conclusion}

India as well as world's environment is becoming fragile and environmental pollution is one of the undesirable side effects of industrialization, urbanization, population growth and unconscious attitude towards the environment. At present, environmental protection is the main need of the society. Though industrialization and development in agriculture are necessary to meet the basic requirement of people, at the same time it is necessary to preserve the environment. In India, too, the environmental pollution has become a cause of concern at various levels. In India, due to lack of sewage treatment plants, generally untreated sewage effluents are released either on agricultural land for irrigation or disposed of in nearby water bodies. In general, sewage effluents from industries and municipal origin contain appreciable amounts of plants nutrients and variable amount of metallic cations like $\mathrm{Hg}, \mathrm{Co}, \mathrm{Zn}, \mathrm{Cu}, \mathrm{Fe}, \mathrm{Mn}$, $\mathrm{Pb}, \mathrm{Ni}, \mathrm{Cd}$, etc. Long-term irrigation with such effluents increases heavy metals accumulation in soils and the chances of their entrance in food chain and this ultimately causes significantly health concern. Thus, it becomes necessary to study the composition of sewage waters and heavy metals accumulation, with the help of advance techniques. The effects of toxic substances on plants are dependent on the amount of toxic substance taken up from the given environment. Germination and seedling 
Table 5. Path coefficient analysis showing direct and indirect effect on seedling vigour in linum usitatissimum L.

\begin{tabular}{|c|c|c|c|c|c|c|c|c|c|}
\hline \multirow[b]{2}{*}{ Character } & \multicolumn{9}{|c|}{ Indirect effect } \\
\hline & $\begin{array}{c}\text { Correlation } \\
\text { with seedling } \\
\text { vigour }\end{array}$ & $\begin{array}{l}\text { Direct } \\
\text { effect }\end{array}$ & $\begin{array}{c}\% \\
\text { Germination }\end{array}$ & $\begin{array}{l}\text { Germination } \\
\text { rate index }\end{array}$ & $\begin{array}{l}\text { Radicle } \\
\text { length }\end{array}$ & $\begin{array}{c}\text { Radicle } \\
\text { elongation } \\
\text { rate }\end{array}$ & $\begin{array}{l}\text { Hypocotyl } \\
\text { length }\end{array}$ & $\begin{array}{c}\text { Hypocotyl } \\
\text { Elongation } \\
\text { Rate }\end{array}$ & $\begin{array}{l}\text { Seedling } \\
\text { length }\end{array}$ \\
\hline \multirow{2}{*}{$\%$ Germination } & $\mathrm{Rg} 0.552$ & $g-7.070$ & \multirow{2}{*}{------- } & 1.728 & -4.229 & 2.204 & -0.843 & -0.052 & 2.945 \\
\hline & Rp0.547 & $\mathrm{p} 0.350$ & & -0.091 & -0.058 & -0.009 & -0.032 & 0.001 & 0.238 \\
\hline \multirow{2}{*}{$\begin{array}{l}\text { Germination } \\
\text { rate index }\end{array}$} & 0.531 & 1.729 & -7.049 & \multirow{2}{*}{----- } & -3.666 & 1.977 & 0.720 & -0.009 & 2.545 \\
\hline & 0.519 & 0.093 & 0.344 & & -0.050 & -0.008 & -0.028 & 0.000 & 0.205 \\
\hline \multirow{2}{*}{$\begin{array}{l}\text { Radicle } \\
\text { length }\end{array}$} & 0.650 & -12.714 & -2.345 & 0.498 & & 6.131 & -2.696 & -0.832 & 9.002 \\
\hline & 0.638 & -0.192 & 0.106 & -0.024 & ------ & -0.030 & -0.118 & 0.023 & 0.805 \\
\hline \multirow{2}{*}{$\begin{array}{l}\text { Radicle elongation } \\
\text { rate }\end{array}$} & 0.679 & 6.259 & -2.483 & 0.518 & -12.455 & & -3.226 & -0.901 & 9.445 \\
\hline & 0.650 & -0.031 & 0.107 & -0.024 & -0.186 & ------- & -0.139 & 0.024 & 0.806 \\
\hline \multirow{2}{*}{ Hypocotyl length } & 0.709 & -12.071 & -0.492 & 0.103 & -2.840 & 1.672 & & -2.162 & 14.416 \\
\hline & 0.690 & -0.533 & 0.021 & -0.005 & -0.043 & -0.008 & ----------- & 0.060 & 1.294 \\
\hline \multirow{2}{*}{$\begin{array}{l}\text { Hypocotyl } \\
\text { elongation } \\
\text { rate }\end{array}$} & 0.714 & 2.228 & -0.166 & 0.007 & -4.748 & 2.530 & -11.715 & & 14.941 \\
\hline & 0.699 & 0.062 & 0.005 & 0.000 & -0.070 & -0.012 & -0.514 & -------- & 1.331 \\
\hline \multirow{2}{*}{ Seedling length } & 0.723 & 15.588 & -1.333 & 0.282 & -7.342 & 3.792 & -11.163 & -2.136 & \\
\hline & 0.701 & 1.400 & 0.060 & -0.014 & -0.111 & -0.018 & -0.492 & 0.059 & ------- \\
\hline \multirow{2}{*}{ Cotyledonary area } & 0.176 & 1.361 & 2.688 & -0.660 & 1.200 & -0.808 & -6.452 & -1.328 & 6.414 \\
\hline & 0.163 & -0.038 & -0.127 & 0.033 & 0.017 & 0.004 & -0.279 & 0.036 & 0.567 \\
\hline \multirow{2}{*}{$\begin{array}{l}\text { Seedling fresh } \\
\text { weight }\end{array}$} & 0.435 & 89.512 & 2.153 & -0.621 & -7.940 & 3.829 & -5.779 & -1.306 & 10.054 \\
\hline & 0.429 & -1.363 & -0.102 & 0.032 & -0.119 & -0.018 & -0.254 & 0.036 & 0.900 \\
\hline \multirow{2}{*}{$\begin{array}{l}\text { Seedling dry } \\
\text { Weight }\end{array}$} & 0.437 & -12.113 & 2.038 & -0.601 & -8.241 & 4.035 & -5.488 & -1.216 & 9.882 \\
\hline & 0.419 & 0.049 & -0.093 & 0.031 & -0.119 & -0.018 & -0.233 & 0.033 & 0.854 \\
\hline \multirow{2}{*}{$\begin{array}{l}\text { Absolute Seedling } \\
\text { Water content }\end{array}$} & 0.434 & -91.484 & 2.156 & -0.621 & -7.916 & 3.815 & -5.786 & -1.308 & 10.050 \\
\hline & 0.428 & 1.012 & 0.102 & 0.032 & -0.119 & -0.018 & -0.255 & 0.036 & 0.900 \\
\hline \multirow{2}{*}{$\begin{array}{l}\text { Specific Seedling } \\
\text { Water Content }\end{array}$} & 0.273 & 2.412 & 0.697 & -0.166 & -2.747 & 1.099 & -4.340 & -1.017 & 6.016 \\
\hline & 0.209 & 0.112 & 0.027 & 0.003 & -0.034 & -0.005 & -0.152 & 0.021 & 0.431 \\
\hline \multirow{2}{*}{$\begin{array}{l}\text { Seed Vigour } \\
\text { index }\end{array}$} & 0.682 & 15.131 & 0.625 & 0.053 & -10.189 & 5.028 & -6.250 & -1.313 & 11.643 \\
\hline & 0.660 & 0.303 & 0.041 & -0.004 & -0.146 & -0.023 & -0.263 & 0.035 & 0.996 \\
\hline \multirow{2}{*}{ Seed Size } & 0.005 & -0.030 & 3.396 & -0.0874 & -3.409 & 1.406 & -1.948 & -0.655 & 3.738 \\
\hline & -0.004 & -0.001 & 0.142 & 0.036 & -0.044 & -0.006 & -0.073 & 0.015 & 0.286 \\
\hline \multirow{2}{*}{1000 Seed weight } & 0.020 & -2.096 & 3.732 & -0.964 & 0.076 & 0.194 & -4.320 & -0.802 & 4.637 \\
\hline & 0.067 & -0.039 & 0.173 & 0.047 & 0.001 & -0.001 & -0.190 & 0.022 & 0.415 \\
\hline
\end{tabular}


Table 5. Continued.

\begin{tabular}{|c|c|c|c|c|c|c|c|c|}
\hline \multirow[b]{2}{*}{ Character } & \multicolumn{8}{|c|}{ Indirect effect } \\
\hline & $\begin{array}{l}\text { Cotyledonary } \\
\text { area }\end{array}$ & $\begin{array}{l}\text { Seedling } \\
\text { fresh } \\
\text { weight } \\
\end{array}$ & $\begin{array}{c}\text { Seedling } \\
\text { dry } \\
\text { weight } \\
\end{array}$ & $\begin{array}{l}\text { Absolute seedling } \\
\text { water content }\end{array}$ & $\begin{array}{c}\text { Specific seedling } \\
\text { water } \\
\text { content } \\
\end{array}$ & $\begin{array}{c}\text { Seedling } \\
\text { vigour } \\
\text { index } \\
\end{array}$ & $\begin{array}{l}\text { Seed } \\
\text { size }\end{array}$ & $\begin{array}{l}1000 \text { seed } \\
\text { weight }\end{array}$ \\
\hline \multirow{2}{*}{$\%$ Germination } & -0.519 & -27.335 & 3.502 & 27.973 & -0.238 & 1.341 & 0.014 & 1.110 \\
\hline & 0.014 & 0.396 & -0.013 & -0.294 & -0.009 & 0.036 & 0.000 & 0.019 \\
\hline \multirow{2}{*}{$\begin{array}{l}\text { Germination } \\
\text { rate index }\end{array}$} & -0.520 & -32.164 & 4.213 & 32.883 & -0.232 & 0.460 & 0.015 & 1.169 \\
\hline & 0.014 & 0.464 & -0.016 & -0.344 & -0.004 & 0.015 & 0.000 & 0.020 \\
\hline \multirow{2}{*}{ Radicle length } & 0.128 & 55.898 & -7.852 & -56.963 & 0.521 & 12.125 & -0.008 & 0.013 \\
\hline & 0.003 & -0.847 & 0.030 & 0.627 & 0.020 & 0.230 & 0.000 & 0.000 \\
\hline \multirow{2}{*}{$\begin{array}{l}\text { Radicle elongation } \\
\text { rate }\end{array}$} & -0.176 & 54.767 & -7.809 & -55.796 & 0.423 & 12.156 & -0.007 & -0.065 \\
\hline & 0.004 & -0.815 & 0.030 & 0.603 & 0.018 & 0.224 & 0.000 & -0.001 \\
\hline \multirow{2}{*}{ Hypocotyl length } & 0.727 & 42.854 & -5.507 & -43.849 & 0.867 & 7.835 & -0.005 & -0.750 \\
\hline & -0.020 & -0.651 & 0.021 & 0.484 & 0.032 & 0.150 & 0.000 & -0.014 \\
\hline \multirow{2}{*}{$\begin{array}{l}\text { Hypocotyl } \\
\text { elongation } \\
\text { rate }\end{array}$} & 0.811 & 52.452 & -6.611 & -53.715 & 1.101 & 8.917 & -0.009 & -0.755 \\
\hline & -0.022 & -0.790 & 0.026 & 0.588 & 0.038 & 0.172 & 0.000 & -0.014 \\
\hline \multirow{2}{*}{ Seedling length } & 0.560 & 57.732 & -7.679 & -58.982 & 0.931 & 11.302 & -0.007 & -0.623 \\
\hline & -0.015 & -0.877 & 0.030 & 0.651 & 0.035 & 0.216 & 0.000 & -0.012 \\
\hline \multirow{2}{*}{ Cotyledonary area } & & 40.121 & -3.628 & -41.587 & 1.704 & 2.507 & 0.021 & -1.333 \\
\hline & ------ & -0.694 & 0.014 & 0.447 & 0.062 & 0.046 & 0.000 & -0.024 \\
\hline \multirow{2}{*}{$\begin{array}{l}\text { Seedling fresh } \\
\text { weight }\end{array}$} & 0.610 & & -11.820 & -91.480 & 1.148 & 13.536 & -0.022 & -1.440 \\
\hline & -0.016 & ------- & 0.047 & 1.012 & 0.040 & 0.262 & 0.000 & -0.027 \\
\hline \multirow{2}{*}{ Seedling dry Weight } & 0.408 & 67.344 & & -89.062 & 0.659 & 14.033 & -0.015 & -1.224 \\
\hline & -0.011 & 1.296 & -------- & 0.958 & 0.006 & 0.280 & 0.000 & -0.022 \\
\hline \multirow{2}{*}{$\begin{array}{l}\text { Absolute Seedling } \\
\text { Water content }\end{array}$} & 0.619 & 39.508 & -11.793 & & 1.169 & 13.497 & -0.022 & -1.448 \\
\hline & -0.017 & -1.363 & 0.046 & ------- & 0.042 & 0.260 & 0.000 & 0.027 \\
\hline \multirow{2}{*}{$\begin{array}{l}\text { Specific Seedling } \\
\text { Water Content }\end{array}$} & 0.962 & 42.597 & -3.308 & -44.342 & & 3.707 & -0.029 & -1.255 \\
\hline & -0.021 & 0.486 & 0.003 & 0.375 & ------ & 0.008 & 0.000 & 0.019 \\
\hline \multirow{2}{*}{ Seed Vigour index } & 0.225 & 30.074 & -11.234 & -81.602 & 0.591 & & -0.010 & -0.839 \\
\hline & -0.006 & -1.176 & 0.045 & 0.868 & 0.003 & -------- & 0.000 & -0.015 \\
\hline \multirow{2}{*}{ Seed Size } & 0.982 & 64.890 & -6.380 & -67.082 & 2.396 & 5.327 & & -1.751 \\
\hline & -0.021 & -0.811 & 0.022 & 0.608 & 0.062 & 0.091 & ------ & -0.025 \\
\hline \multirow{2}{*}{1000 Seed weight } & 0.866 & 61.497 & -7.074 & -63.214 & 1.455 & 6.059 & -0.025 & \\
\hline & -0.023 & -0.934 & 0.028 & 0.698 & 0.055 & 0.115 & 0.000 & -------- \\
\hline
\end{tabular}

Residual effect (genotypic) $=-0.0278$; residual effect $($ phenotypic $)=-0.0078$. 
establishment are vulnerable stages in the plant life cycle.Seedling growth is considered as an indicator of metal stress on plant ability to survive. The seed germination and seedling establishment is notoriously sensitive to heavy metals often a difference i.e., a noticeable change in growth rate. There may be a specific gene in plants which monitor the toxicity level. This gene is responsible for the different strength of tolerance to a variety of toxic compound in different plant species or varieties. The present study emphases the importance of several parameters for assessment of the comparative resistance of cultivars of a crop species. The evaluation of germination and seedling growth may be helpful in assessing the relative resistance or susceptibility of cultivars to heavy metals for cultivation would be meaningful in minimizing the heavy metals effect. This study is crucial to be delivery of the potential of possible loss in yield beyond the trials and research farms to agriculture in general, because crop performance is entirely determined by a complex genotype and environment interaction.

\section{ACKNOWLEDGEMENT}

Authors wish to acknowledge the principal scientist, Division of Plant Breeding and Genetics, C.S. Azad University of Agriculture and Technology, Kanpur, India for supplying the seed of various cultivars of Linum usitatissimum $L$.

\section{REFERENCES}

Abdulbaki AA, Anderson JD (1973). Vigour determination in soybean seed by multiple criteria. Crop Sci. 13:630-633.

Allard RW (1960). Principal of Plant Breeding (ed) John Wiley \&Sons Inc.London.

Ayaz F.A. Kadioglu A (1997). Effects of heavy metals (Zn, Cd, Cu, Hg) on the soluble protein bands of germinating Lens esculenta $\mathrm{L}$. seeds. Turk. J. Bot. 21:85-88.

Aydinlap C Marinova S (2009). The effects of heavy metals on seed germination and plant growth on alfalfa plant (Medicago sativa). Bulg. J. Agric. Sci. 15:347-350.

Azevedo RA, Lea PJ (2005). Toxic metals in plants. Braz. J. Plant Physiol. 17:1.

Baker AJM (1987). Metal tolerance. New Phytol. 106:93-111.

Baker AJ, Proctor J (1990). The influence of $\mathrm{Cd}, \mathrm{Cu}, \mathrm{Pb}$ and $\mathrm{Zn}$ on the distribution and evolution of metallophytes in British island. Plant. Syst. Evol. 173:91-108.

Basak M, Sharma M, Chakraborty U (2001). Biochemical response of Camellia sinensis to heavy metal stress. J. Environ. Biol. 22(1):3741.

Bates GR (1940). Growth behavior of plants following seed treatment by organic mercury compounds. Nature 145:262-263.

Benavides MP, Gallego SM, Tomaro ML (2005). Cadmium toxicity in plants. Braz. J. Plant Physiol. 17:21-34.

Blaylock AD, Davis TD, Jolley VD, Walser RH (1982). Influence of Cobalt and Iron on photosynthesis, Chlorophyll and nutrient content in regreening Chlorotic tomatoes and soybeans. J. Plant Nutr. 9:823838.

Blaylock MJJ, Huang W (2000). Phytoextraction of metals In: I. Raskin and B.D. Ensley (Ed.) Phytoremediation. Proceedings of the 2000
Conference on Hazardous Waste Research, p. 139.

Boyajian GEL, Carreira H (1997). Phytoremediation: a clean transition from laboratory to marketplace. Nat. Biotechnol. 15:127-128.

Bradshaw AD (1984). Adaptations of plants to sails containing toxic metals. A test for conceit In: Evered Dand, G.M. Collins (ed.) Origin and Development of Adaptation. Ciber Found. Symp. 102:4-19, Pitman U.K.

Bradshaw AD (1991). Genostasis and the limits to evolution. Philosophical transaction of the Royal society of London. Series B 333:289-305.

Brenchley WE (1938). Comparative effects of Cobalt, Nickel and Copper on plant growth. Ann. Appl. Biol. 25:671-694.

Brown MT, Wilkins DA (1986). The effect of zinc on germination, survival and growth of Betula seeds. Environ. Pollut. Ser. A. 41(1):5361.

Burton GW (1952). Quantitative inheritance in grasses. Proceeding VI Institute Grassland Congress; I: 409-417.

Carleton AE Cooper CS (1972). Seed size effect upon the seedling vigour of three legumes. Crop Sci. 12:176-177.

Chatterjee J, Chatterjee C (2000). Phytotoxicities of cobalt and copper in cauliflower. Environ. Pollut. 109(1):69-74.

Claire LC Adriano DC, Sajwan KS, Abel SL, Thoma DP Driver JT (1991). Effects of selected trace metals on germinating seeds of six plant species. Water Air Soil Pollut. 59:231-240.

Coughtrey PJ, Jones CH Martin MH (1979). Cd, Pb and Zn interactions and tolerance in two populations of Holcus lanatus $L$. grown in solution culture. Environ. Exp. Bot. 19:285-290.

Cunningham SD, Shann JR, Crowley DE, Anderson TA (1997). Phytoremediation of contaminated water and soil. Phytoremediation of soil and water contaminants. American Chemical Society, Washington, DC pp. 2-17

De Vos CHR, Schat H, De Waal MAM, Voojs R, Ernst WHO (1991). Increase resistance to copper induced damage of root cell plasmalemma in copper tolerant Silene cucubalus. Plant Physiol. 82:523-528.

Dewey JR, Lu KH (1959). A correlation and path coefficient analysis of components of creasted wheat grass (A gropyron cristatum) seed production. Agron. J. 51:515-518.

Dickinson NM, Turner AP, Lepp NW (1991). Survival of trees in a metal contaminated environment. Water, Air Soil Pollut. 57-58:627-633.

Dueck TA, Wotting HG, Moet DR, Pasman FJM (1987). Growth and reproduction of Silene cucubalis. W/B intermittently exposed to low concentration of pollutants Zn, Cu. New Phytol. 105:633-645.

Dushenkov S, Kapulnik Y, Blaylock M, Sorochisky B, Raskin I, Ensley B (1997). Phytoremediation: a novel approach to an old problem. Global environmental biotechnology proceedings of the Third Biennia Meeting of the International Society for Environmental MA, Elsevier, New York, p. 563.

Ebbs SD, Kochian LV (1997). Toxicity of zinc and copper to Brassica species: Implications for Phytoremediation. J. Environ. Qual. 5:776781.

Ebbs SD, Lasat MM, Brady DJ, Cornish J, Gordon R, Kochian LV (1997). Phytoextraction of cadmium and zinc from contaminated soil. J. Environ. Qual. 26:1424-1430.

Eberhard SA, Russell WA (1966). Stability parameters for comparing varieties. Crop Sci. 6:36-40.

Eduardo MJ, Jesús MP, Elvira E, Ramón O, Carpena R (2007) .Mercury accumulation and resistance to mercury stress in Rumex induratus and Marrubium vulgare grown in perlite. J. Plant Nutr. Soil Sci. 170(4):485-494

Enger M, Campbell E, Raflif R (1997). Cadmium induced alteration in RNA metabolism in culture of Chinese hamster cells sensitive and resistant to the cytotoxic effects of cadmium. J. Toxicol. Environ. Health 5:711-728.

Fernandez JC, Henriquez FS (1991). Biochemical, physiological and structural effect of excess copper in plants. Bot. Rev. 57:246-273.

Garcia JS, de Magalhães CS Arruda MAZ (2006). Trends in metalbinding and metalloprotein analysis. Talanta 69:1-15.

Gardea-Torresdey JL, Polette L, Arteaga A, Tiemann KJ, Bibb J, Gonzalez JH (1996). Determination of the content of hazardous heavy metals on Larrea tridentata grown around a contaminated area. Proceedings of the Eleventh Annual EPA Conf. On Hazardous 
waste Research, Edited by Erickson LR, Tillison DL, Grant SC, McDonald JP, Albuquerque NM, p. 660.

Gartside DW, McNeilly TB (1974). The potential for evolution of heavy metal tolerance in plant II. Cu tolerance in normal populations of different plant species. Heredity 32:335-348.

Gelmond H (1972). Relationship between seed size and seedling vigour in Cotton (Gossypium hirsutum). Proceeding International Seed Test Association, 37:3.

Gomes-Junior RA, Moldes CA, Delite FS, Pompeu GB, Gratão PL, Mazzafera P, Lea PJ, Azevedo RA (2006). Antioxidant metabolism of coffee cell suspension cultures in response to cadmium. Chemosphere 65:1330-1337.

Gratão PL, Polle A, Lea PJ Azevedo RA (2005a). Making the life of heavy metal-stressed plants a little easier. Funct. Plant Biol. 32:481494.

Gratão PL, Prasad MNV, Cardoso F, Lea PJ, Azevedo RA (2005b). Phytoremediation: green technology for the clean up of toxic metals in the environment. Braz. J. Plant Physiol. 17:53-64.

Haselhoff $E$ (1895). Experiments on injurious action on plants of water containing cobalt. Landw Johrb. 24:959-961.

Heydecker W (1973). Glossary of Terms in Seed Ecology (ed.). pp. 553-557. Bulten Worts. London.

Huillier LL, Auzac JD, Durand M, Michaud-Ferriere N (1996). Nickel effects on two maize (Zea mays) cultivars: growth, structure, $\mathrm{Ni}$ concentration, and localization. Can. J. Bot. 74:1547-1554.

Hussaini SH, Sarada P, Reddy BM (1984). Effect of seed size on germination and vigour in maize. Seed Res. 12:98-101.

Hutchirison TC (1984). Adaptation of plants atmospheric pollutants In: Evered, D. and G.M. Collins (ed) Origin and Development of Adaptation pp. 52-57. Pitman London.

International seed testing association (1976). International Rules for Seed Testing. Seed Sci. Technol. 4:349.

lqbal MZ, Mehmood T (1991). Influence of cadmium toxicity on germination and growth of some common trees. Pak. J. Sci. Ind. Res. $34: 140-142$

Jamal SN, Iqbal Z, Athar M (2006). Evaluation of two wheat varieties for phytotoxic effect of mercury on seed germination and seedling growth. Agriculture Cienconspectus Sticus (ACS). 71(2):41-44.

Jarup $L$ (2003). Hazard of heavy metal contamination. Br. Med. Bull. 68:167-182

Jiang W, Liu D Li MX (1994). Effects of Cd on the nucleolus in root tip cells of Allium cepa. J. Environ. Sci. 6:382-386.

Jiang W, Liu D Liu X (2001). Effects of copper on root growth, cell division, and nucleolus of Zea mays. Biol. Plant 44(1):105-109.

Johanson HW, Robinson HF, Comstock RE (1955). Estimates of genetic and environmental variability in soybean. Agron. J. 47:314318.

Kabir M, lqbal MZ, Shafigh M, Faroogi ZR (2008). Reduction in germination and seedling growth of Thespesia populnea $\mathrm{L}$. caused by lead and cadmium treatments. Pak. J. Bot. 40(6):2419-2426.

Kulkarni RG, Nayeem GA (1986). Effect of temperature on seed germination and seedling vigour index in wheat. Paper presented at 21st I.S.T. A Symposium held at Brisbane (Australia) from 14-18th July 1986.

Li XL, Zheng GR (1992). Study on mutagenic effects and antagonism of selenium and cadmium. Carcinogenesis, Teratogenesis Mutagenesis 4:19-21.

Liu D, Jiang W Gao X (2003). Effect of cadmium on root growth, cell division and nucleoli in root tip cells of garlic. Biol. Plant 47(1):79-83.

Lynch JM, Moffat AJ (2005). Bioremediation - prospects for the future application of innovative applied biological research. Ann. Appl. Biol. 146:217-222.

Mahmood S, Hussain A, Saeed, Z Athar M 2005. Germination and seedling growth of corn (Zea mays L.) under varying levels of copper and zinc. Int. J. Environ. Sci. Technol. 2:269- 274.

Mcnair MR (1990). The genetic of metal tolerance in natural population. In: Shaw, A.J. (ed.) Heavy Metal Tolerance in Plants Evolutionary Aspects pp. 235-255.

Meagher RB (2000). Phytoremediation of toxic elemental and organic pollutants.C. Op. Plant Biol. 3:153-162.

Miller RR (1996). Phytoremediation: technology overview report. Ground Water Remediation Technologies Analysis.140 Proceedings of the 2000 Conference on Hazardous Waste Research.

Mitchell RL (1945). Cobalt and Nickel in soils and plants. Soil Sci. 60:63-67.

Moffat AS (1995). Plants proving their worth in toxic metal. Science 269:302-303.

Morzeck JRE, Funicelli NA (1982). Effect of zinc and lead on germination of Spartina alterniflora Loisel., seeds at various salinities. Environ. Exp. Bot 22:23-32.

Mulett JH, Wilkinson RI (1979). The relationship between amount of electrolytes loss on leaching in seed of Pisum sativum and some parameters of plant growth. Seed Sci. Technol. 7:381-398.

Nanda PBA, Dushenkov V, Motto H, Raskin I (1995). Phytoextraction: the use of plants to remove heavy metals from soil. Environ. Sci. Technol. 29:1232-1238.

Nayeem KA, Deshpande US (1987). Genetic variability and correlation coefficient relating to seed size, seedling vigour and some physiochemical properties in wheat. Seed Sci. Technol. 15:699-704.

Palit S, Sharma A, Talukder G (2008). Effect of Cobalt on plants. Bot. Rev.1874-9372(Online) pp. 149-181.

Panse VG, Sukhatme PV (1961). Statistical method for Agricultural workers (ed) I.C.A.R. New Delhi.

Pilon-Smits E A H 2005. Phytoremediation. Ann. Rev. Plant Biol. 56:1539.

Radha J, Srivastava S, Solomon S, Shrivastava A K Chandra A (2010). Impact of excess zinc on growth parameters, cell division, nutrient accumulation, photosynthetic pigments and oxidative stress of sugarcane (Saccharum spp.). Acta Physiol. Plant. 32:979-986.

Rahman KM, Khan M (2010). Effect of varying concentration of nickel and cobalt on the plant growth and yield of chickpea. Aust. J. Basic Appl. Sci. 4(6):1036-1046.

Raskin I, Ensley BD (2000). Phytoremediation of toxic metals: using plants to clean up the environment (ed) John Wiley and Sons, New York, p. 303

Reddy T Vaidyanath K (1978). Mutagenic potentiating and antimutagenic activity of certain metallic ions in the rice genetic system. Curr. Sci. 47:513-515.

Robinson HF (1966). Quantitative genetics in relation to breeding on centennial of Mendalism. Indian J. Genet. 26:171-187.

Searle SR (1961). Phenotypic, genotypic and environmental correlation. Biometrics 17:474-480.

Shafiq M, lqbal MZ (2005). The toxicity effects of heavy metals on germination and seedling growth of Cassia siamea Lamk. J. New Seeds 7:95-105.

Shafiq M, lqbal MZ, Athar M (2008). Effect of lead and cadmium on germination and seedling growth of Leucaena leucocephala. J. Appl. Sci. Environ. Manag. 12(2):61-66.

Sharma SS (1984). Differential response of Cucurbits cultivars to mercury pretreatment on germination and seedling growth. Seed Res. (New Delhi) 12:65-69.

Simmonds NW (1962). Variability in crop plants, its use and conservation. Biol. Rev. 37:422-465.

Simmonds NW (1979). Principles of Crop Improvement (ed.) Longman London.

Singh D, Nath K, Sharma YK (2007). Response of wheat seed germination and seedling growth under copper stress. J. Environ. Biol. 28(2):409-414

Taulavuori K, Prasad MN, Taulavuori E, Laine K (2005). Metal stress consequences on frost hardiness of plants at northern high latitudes: a review and hypothesis. Environ. Pollut. 135:209-220.

Tkachuk R, Kuzina FD (1972). Mercury level in wheat and other cereals, oilseeds and biological samples. J. Food Agric. Sci. 23:11831195.

Vergano O, Hunter JG (1952). Nickel and Cobalt toxicities in oat plants. Ann. Bot. 17:317-328.

Wasay SA, Barrington SF, Tokunaga S (1998). Using Aspergillus niger to biorremediate soils contaminated by heavy metals. Biorem. J. 2(3):183-190

Wilkins DA (1978). The measurement of tolerance of edaphic factors by means of root growth. New Phytol. 80:623-633.

Xu YJ, Huystee RB, Huystee V (1993). Effect of Ca, its inhibitor and heavy metals on growth cycle of peanut cell aggregates. Plant Cell Tissue Organ Cult. 32:319-328. 
Yang GF, Li Q, Zhang CG, Shang SH, Jing QN, Xu HX (1986). The effect of lead cadmium mineral oil in soil microorganism and soil enzyme. In Xia ZL Editor and Study of Soil Environmental Capacity. Meteorological Publishing House, Beijing, pp. 157-164.
Zhang Y, Yang X (1994). The toxic effects of cadmium on cell division and chromosomal morphology of Hordeum vulgare. Mutat. Res. 312:121-126. 